\title{
Gaussian Fluctuations of Two Molecular Fields in the Blume-Emery-Griffiths Model
}

\author{
W. JAWORSKI AND Z. JACYNA-ONYSZKIEWICZ \\ Quantum Physics Division, Faculty of Physics, Adam Mickiewicz University \\ Umultowska 85, 61-614 Poznań, Poland
}

(Received October 6, 2010; in final form January 5, 2011)

\begin{abstract}
The Blume-Emery-Griffiths model for spins $S=1$ in a bilayer with $z=5$ nearest neighbours is studied with the use of Gaussian fluctuations approximation. The fluctuations of two molecular fields, connected with two order parameters, are introduced. Their influence on phase diagrams for non-negative values of the biquadratic coupling constant is taken under consideration. The results are compared with those obtained by the mean-field approximation and discussed.
\end{abstract}

PACS: 75.10.Dg, 75.10.Hk, 75.10.Jm

\section{Introduction}

The Blume-Emery-Griffiths (BEG) model introduced for describing the thermodynamics of ${ }^{3} \mathrm{He}-{ }^{4} \mathrm{He}$ mixtures [1] has been studied for more than thirty years by now. However, the simply looking spin-1 Ising Hamiltonian has attracted physicists dealing not only with helium mixtures or magnets but a vast variety of systems like fluid and liquid crystal mixtures or semiconductor alloys. Furthermore, the rich phase diagrams exhibited by the BEG model has been the field for testing many calculation (mean field approximation [1, 2], renormalization group theory [3-5]) and simulation (Monte Carlo methods [6-9]) techniques. Some lattice types, like the honeycomb [10] or the Bethe lattice [11] allowed to obtain exact solutions.

More information on the BEG model itself as well as on transforming it into the extended Hubbard model may be found in some recent works by Mancini et al. [12, 13]. Many interesting results were also given by the cluster variational method in pair approximation (CVMPA) $[14,15]$. For example, the case of a bilayer with $z=5$ nearest neighbours was studied in the whole range of parameters [16].

In this paper however we consider only ferromagnetic bilinear $(J>0)$ and biquadratic $(A>0)$ interactions and no magnetic field $(h=0)$. We show that fluctuations of two molecular fields derived within the Gaussian fluctuations approximation (GFA) make the phase diagrams of the BEG model differ significantly from those obtained in the mean-field approximation (MFA).

The methodology of the Gaussian fluctuations approximation is presented in Sect. 2 and in Sect. 3 the results are presented and discussed.

\section{Calculations}

The BEG model is described by the spin- $1\left(\sigma_{i}=\right.$ $-1,0,1)$ Ising Hamiltonian including the bilinear $(J)$ and biquadratic $(A)$ nearest-neighbour $(z=5)$ interactions, as well as the magnetic field $(h)$ and single-ion anisotropy $(D)$ terms, which may be written in the form

$$
\begin{aligned}
H & =-J \sum_{\langle i, j\rangle} \sigma_{i} \sigma_{j}-h \sum_{i} \sigma_{i}-A \sum_{\langle i, j\rangle} \sigma_{i}^{2} \sigma_{j}^{2} \\
& -D \sum_{i} \sigma_{i}^{2} .
\end{aligned}
$$

Two order parameters being the mean values of the magnetic and quadrupole moment, $m=\left\langle\sigma_{i}\right\rangle$ and $q=\left\langle\sigma_{i}^{2}\right\rangle$, respectively, are derived by expanding the right hand side of

$$
\left\langle\sigma_{i}^{s}\right\rangle=\frac{\operatorname{Tr}\left(\sigma_{i}^{s} \exp \left(-\beta\left(H_{0}+H_{1}\right)\right)\right)}{\operatorname{Tr}\left(\exp \left(-\beta\left(H_{0}+H_{1}\right)\right)\right)},
$$

into a series with respect to the perturbation $H_{1}=$ $H\left(\sigma_{i}^{s} \rightarrow \delta \sigma_{i}^{s}\right)$, where $s \in\{1,2\}$ and $\delta \sigma_{i}^{s}=\sigma_{i}^{s}-\left\langle\sigma_{i}^{s}\right\rangle$ is the fluctuation operator. The mean-field type Hamiltonian $H_{0}=H-H_{1}$ provides us with two molecular fields $y$ and $\zeta$ which will be named the molecular and the quadrupolar field, respectively, for the purpose of that paper. They may be given by

$$
y=\beta(h+J z m), \quad \zeta=\beta(D+A z q),
$$

where $\beta=1 / T$ (with $k_{\mathrm{B}}=1$ ).

By choosing certain partial sums in the infinite series in (2) for $m$ and $q$ we can use the graphical representation presented in [17]:

$$
\begin{aligned}
& m=\left\langle\sigma_{i}\right\rangle=\bullet|\bullet \leftrightarrow \bullet| \cdot \leftrightarrow \bullet, \\
& q=\left\langle\sigma_{i}^{2}\right\rangle=\||\bullet \leftrightarrow \bullet| \cdot \leftrightarrow \bullet .
\end{aligned}
$$

This representation is based on the fact that partial 
derivatives of the function

$$
L(y, \zeta)=\ln (2 \exp \zeta \cosh y+1),
$$

may be pictured in the following way:

$$
L_{\nu}^{n}(y, \zeta) \equiv \frac{\partial^{n}}{\partial y^{n}} \frac{\partial^{\nu}}{\partial \zeta^{\nu}} L(y, \zeta) \equiv n \times \bullet \mid \nu \times \bullet .
$$

With symbols representing magnetic $(\bullet)$ and quadrupole (-) moments, the negated arrows ( $\leftrightarrow$ and $\leftrightarrow$ ) in (4) and (5) stand for the renormalized interaction lines, for the bilinear $(J)$ and the biquadratic $(A)$ interaction, respectively. The self-consistent equations for those interaction lines, similar to the one proposed in [17], may be rewritten using the Fourier transforms $J_{k}$ and $A_{k}$ and their representation given below

$$
\begin{aligned}
& \leftrightarrow \equiv \beta J_{\boldsymbol{k}}=\beta \sum_{j} J_{(i j)} \exp \left(\mathrm{i} \boldsymbol{k} \cdot\left(\boldsymbol{r}_{j}-\boldsymbol{r}_{i}\right)\right), \\
& \Leftrightarrow \equiv \beta A_{\boldsymbol{k}}=\beta \sum_{j} A_{(i j)} \exp \left[\mathrm{i} \boldsymbol{k} \cdot\left(\boldsymbol{r}_{j}-\boldsymbol{r}_{i}\right)\right] .
\end{aligned}
$$

We then are able to sum up the series

$$
\begin{aligned}
& \hline \bullet \leftrightarrow \bullet=\leftrightarrow \leftrightarrow \bullet+|\bullet \leftrightarrow \bullet| \bullet \leftrightarrow \bullet \\
&+\bullet \leftrightarrow \bullet|\bullet \leftrightarrow \bullet| \bullet \leftrightarrow \bullet \\
&+\bullet \leftrightarrow \bullet|\bullet \leftrightarrow \bullet| \bullet \leftrightarrow \bullet \mid \bullet \leftrightarrow \bullet+\ldots,
\end{aligned}
$$

for the bilinear and the analogous one for the biquadratic interaction $(\mathbf{-} \leftrightarrow \mathbf{\bullet})$, to obtain the two renormalized interaction lines in the form

$$
\begin{aligned}
& \leftrightarrow=\sum_{\boldsymbol{k}}\left(\beta J_{\boldsymbol{k}}\right)^{2} \bullet \bullet|\bullet \leftrightarrow \bullet| \cdot \leftrightarrow \cdot \equiv(\delta y)^{2}, \\
& \leftrightarrow=\sum_{\boldsymbol{k}}\left(\beta A_{\boldsymbol{k}}\right)^{2} \bullet \bullet|\bullet \leftrightarrow \bullet| \cdot \leftrightarrow \cdot \equiv(\delta \zeta)^{2},
\end{aligned}
$$

which may now be interpreted as the mean fluctuations of the molecular $(\delta y)$ and the quadrupolar $(\delta \zeta)$ field, respectively.

Analytic expressions for $\delta y$ and $\delta \zeta$ as well as for $m$ and $q$ form a set of self-consistent equations, which is characteristic for the GFA method

$$
\begin{aligned}
m & =\overline{\underline{L_{0}^{1}(y, \zeta)}} \\
q & =\underline{\overline{L_{1}^{0}(y, \zeta)}}, \\
(\delta y)^{2} & =\sum_{\boldsymbol{k}}\left(\beta J_{\boldsymbol{k}}\right)^{2} \overline{\underline{L_{0}^{2}(y, \zeta)}} \\
(\delta \zeta)^{2} & =\sum_{\boldsymbol{k}}\left(\beta A_{\boldsymbol{k}}\right)^{2} \underline{\underline{L_{2}^{0}(y, \zeta)}},
\end{aligned}
$$

where $\ldots$ denotes an average over Gaussian distributions of the fields $y$ and $\zeta$. The averaging is made in the way introduced in [17] and used in [18, 19]:

$$
\begin{aligned}
& \frac{\overline{L_{\nu}^{n}(y, \zeta)}}{2 \pi}=\frac{1}{2 \pi} \int_{-\infty}^{\infty} \mathrm{d} x \int_{-\infty}^{\infty} \mathrm{d} \xi \exp \left(-\frac{x^{2}+\xi^{2}}{2}\right) \\
& \quad \times L_{\nu}^{n}(y+x \delta y, \zeta+\xi \delta \zeta) .
\end{aligned}
$$

However, for the purpose of this paper the averaging over the molecular field $\overline{L_{\nu}^{n}(y, \zeta)}$ and the quadrupolar field $L_{\nu}^{n}(y, \zeta)$ are used separately for comparison. For

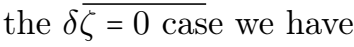

$$
\begin{aligned}
& m=\overline{\bullet \mid \bullet \leftrightarrow \bullet}=\overline{L_{0}^{1}(y, \zeta)}, \\
& q=\overline{\bullet \mid \bullet \leftrightarrow \bullet \bullet}=\overline{L_{1}^{0}(y, \zeta)}, \\
& (\delta y)^{2}=\sum_{\boldsymbol{k}}\left(\beta J_{\boldsymbol{k}}\right)^{2} \overline{\bullet \bullet \mid \bullet \leftrightarrow \bullet} \\
& \quad=\sum_{\boldsymbol{k}}\left(\beta J_{\boldsymbol{k}}\right)^{2} \overline{L_{0}^{2}(y, \zeta)},
\end{aligned}
$$

where

$$
\begin{aligned}
& \overline{L_{\nu}^{n}(y, \zeta)}=\frac{1}{\sqrt{2 \pi}} \int_{-\infty}^{\infty} \mathrm{d} x \exp \left(-\frac{x^{2}}{2}\right) \\
& \times L_{\nu}^{n}(y+x \delta y, \zeta),
\end{aligned}
$$

and for the $\delta y=0$ case

$$
\begin{aligned}
& m=\bullet \mid \cdot \leftrightarrow \bullet=\underline{L_{0}^{1}(y, \zeta)}, \\
& q=\mathbf{- |} \Leftrightarrow \mathbf{-}=\underline{L_{1}^{0}(y, \zeta)},
\end{aligned}
$$

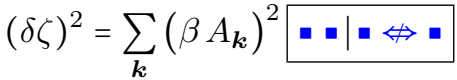

$$
\begin{aligned}
& =\sum_{\boldsymbol{k}}\left(\beta A_{\boldsymbol{k}}\right)^{2} \underline{L_{2}^{0}(y, \zeta)},
\end{aligned}
$$

where

$$
\begin{aligned}
& \frac{L_{\nu}^{n}(y, \zeta)}{\sqrt{2 \pi}} \int_{-\infty}^{\infty} \mathrm{d} \xi \exp \left(-\frac{\xi^{2}}{2}\right) \\
& \quad \times L_{\nu}^{n}(y, \zeta+\xi \delta \zeta) .
\end{aligned}
$$

In each of the three cases given by (13)-(16), (18)-(20) and $(22)-(24)$ an expression for the free energy $(f)$ may be found from the conditions below

$$
\lim _{\delta y, \delta \zeta \rightarrow 0} f=f_{\mathrm{MFA}}
$$

where $f_{\mathrm{MFA}}$ is the free energy obtained within the MFA method and

$$
\frac{\partial f}{\partial m}=0, \quad \frac{\partial f}{\partial q}=0, \quad \frac{\partial f}{\partial \delta y}=0, \quad \frac{\partial f}{\partial \delta \zeta}=0 .
$$

Using the necessary conditions allows us to write the free energy in the following form for the $\delta y \neq 0 \neq \delta \zeta$ case:

$$
\begin{aligned}
\bar{f} & =\frac{z}{2}\left(J m^{2}+A q^{2}\right)-\frac{1}{2 \beta} \overline{\underline{L_{0}^{0}(y, \zeta)}}+\frac{1}{16 J^{2} \beta^{3}}(\delta y)^{4} \\
& +\frac{1}{16 A^{2} \beta^{3}}(\delta \zeta)^{4},
\end{aligned}
$$

the $\delta \zeta=0$ case

$$
\bar{f}=\frac{z}{2}\left(J m^{2}+A q^{2}\right)-\frac{1}{2 \beta} \overline{L_{0}^{0}(y, \zeta)}+\frac{1}{16 J^{2} \beta^{3}}(\delta y)^{4},
$$

and the $\delta y=0$ case

$$
\underline{f}=\frac{z}{2}\left(J m^{2}+A q^{2}\right)-\frac{1}{2 \beta} L_{0}^{0}(y, \zeta)+\frac{1}{16 A^{2} \beta^{3}}(\delta \zeta)^{4} .
$$

Employing each set of formulae given by (13)-(16) and (28), (18)-(20) and (29) or (22)-(24) and (30), en- 
ables us to give a full description of the critical behaviour of the BEG model within the Gaussian fluctuations approximation, with molecular, quadrupolar and both fields fluctuations included

\section{Results}

The phase diagrams for the BEG model in the specified range of parameters $(J>0, A \neq 0$ and $h=0$ ) (for one sublattice system) show transition lines between the

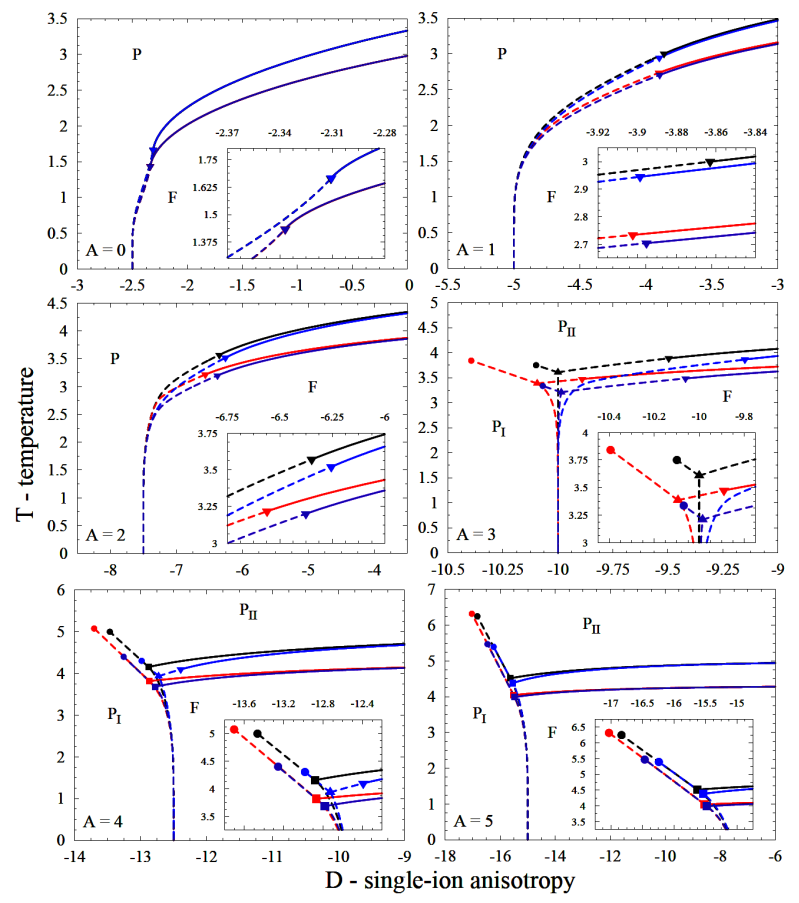

Fig. 1. Phase diagrams of the BEG model on the $D-T$ plane for $J=1, h=0$ and different values of $A=0,1,2,3,4,5$. The lines of second-order transitions are continuous and those of the first kind - discontinuous; the colours represent different levels of approximation used, the marks - different types of critical points and the capital letters - different phases: MFA $\delta y=0=\delta \zeta, \mathbf{G F A}-\delta y \neq 0=\delta \zeta, \mathbf{G F A}-\delta y=0 \neq \delta \zeta$, GFA - $\delta y \neq 0 \neq \delta \zeta ; \bullet-\mathrm{CP},-\mathrm{CEP}, \boldsymbol{\nabla}-\mathrm{TCP}$, $\boldsymbol{\Delta}$ - TP; F - ferromagnetic phase $(m \neq 0 \neq q), \mathrm{P}-$ paramagnetic phase $(m=0)$, subscripts I and II denote different values of the quadrupole moment $(q)$. The insets show parts of the diagrams in a larger scale.

ferromagnetic phase, characterized by $m>0$ and $q>0$, and paramagnetic phase, characterized by $m=0$, and between paramagnetic phases with different values of the quadrupole moment $q$. The lines describing transitions of the first kind (discontinuous) and of the second kind (continuous), as well as the characteristic critical points (CP), critical endpoints (CEP), tricritical points (TCP) and triple points (TP), are obtained within the MFA and three different levels of the GFA. The resulting phase diagrams are presented in Fig. 1.

The phase diagrams for $A=0,3,5$ have already been presented in many papers $[1-4,16]$. The case with no biquadratic interaction $(A=0)$, corresponding to the so-called Blume-Capel model [20], shows no influence of the quadrupolar field fluctuations on transition lines. However, there is a serious effect of the fluctuations of the molecular field, which may be generalised by lowering the temperatures of the continuous ferro-para $(\mathrm{F}-\mathrm{P})$ transitions and the tricritical point. The line of the discontinuous transition on the other hand is almost not affected by any fluctuations, which is due to being in the low temperature region.

The $\mathrm{F}-\mathrm{P}$ transition lines in the diagrams for $A=1,2$ are slightly affected by the fluctuations of the molecular and quadrupolar fields, where the biggest influence is observed for the case with both fluctuations included.

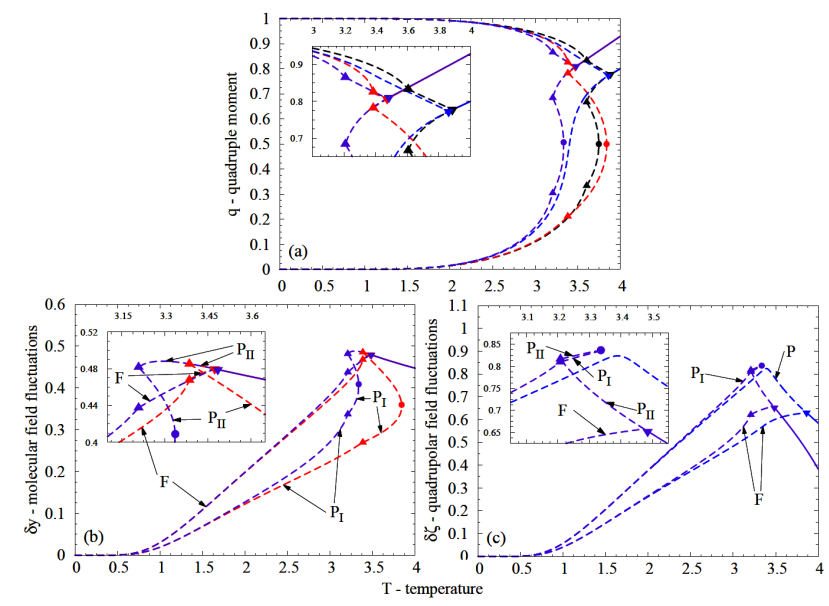

Fig. 2. Plots of the quadrupole moment $q$ (a), the molecular field fluctuations $\delta y(\mathrm{~b})$ and the quadrupolar field fluctuations $\delta \zeta$ (c) along the phase transition lines for $A=3$. The second-order transition lines are continuous. Arrows in (b) and (c) point to regions of the first-order phase transition (discontinuous) lines corresponding to the values obtained in one of the phases F, P, $\mathrm{P}_{\mathrm{I}}$ or $\mathrm{P}_{\mathrm{II}}$ between certain critical points (see Fig. 1 for the symbols and colours). The insets show parts of the plots in a larger scale.

The diagram for $A=3$ exhibits more interesting behaviour including the appearance of two more critical points - CP and TP. The addition of molecular field fluctuations leads to the appearance of temperature-induced magnetism (TIM). The TIM is a well known phenomenon which was already found in the BEG model within the MFA solution but for larger values of the biquadratic coupling $A$. On the other hand, the quadrupolar field fluctuations affect the MFA diagram by erasing the $\mathrm{P}_{\mathrm{I}}-\mathrm{P}_{\mathrm{II}}$ transition line and making it more similar to the diagrams obtained for $A=1,2$. Including the fluctuations of both fields leads to their competition. The behaviour of the quadrupole moment $q$ and the fluctuations $\delta y$ and $\delta \zeta$, plotted along the transition lines (in the way similar to Fig. 2 in [2]), is shown in Fig. $2 \mathrm{a}-\mathrm{c}$.

The regions of high temperature ferromagnetism are present in all the approximation levels for the $A=4$ dia- 
gram. The CP and TP point however are present only in the $\delta y=0$ and $\delta \zeta \neq 0$ case. For the other cases the CEP appears. Temperature dependences of the order parameters, the fluctuations as well as some thermodynamic functions for $D=-6$ are shown in Fig. $3 \mathrm{a}-\mathrm{h}$.

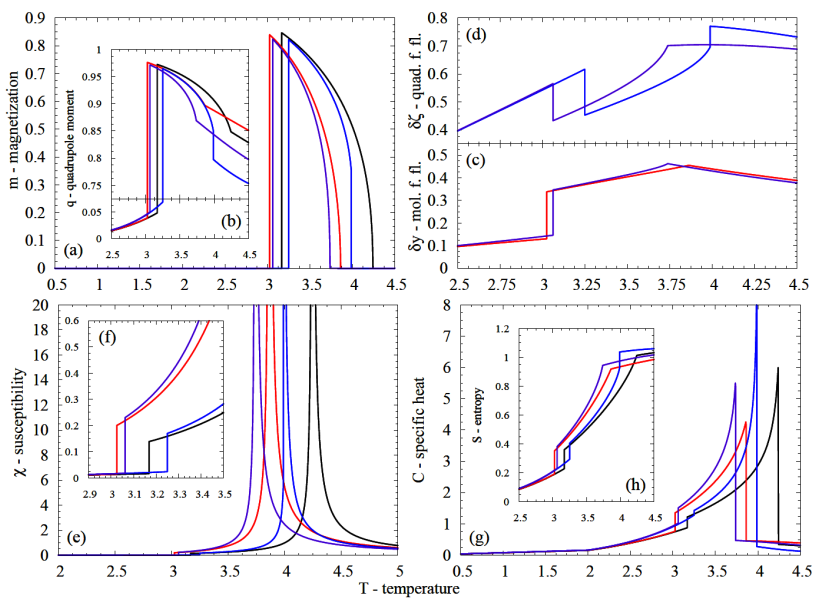

Fig. 3. Plots of the magnetization $m$ (a) and the quadrupole moment $q(\mathrm{~b})$, the molecular $\delta y$ (c) and quadrupolar $\delta \zeta$ (d) field fluctuations, the magnetic susceptibility $\chi(\mathrm{e})$ and (f), the specific heat $C$ (g) and entropy $S$ (h) for $A=4$ and $D=-6$.

The critical endpoint appears in all the phase diagrams for the $A=5$ case. It is again noticeable that the molecular field fluctuations are responsible for lowering the temperatures of the second-order $\mathrm{F}-\mathrm{P}_{\mathrm{II}}$ transition, while the quadrupolar field fluctuations - the temperature of the critical point.

\section{Conclusions}

According to the results we obtained and presented in this paper, the following conclusions can be made.

1. The GFA method allows to include fluctuations in a rather simple way, even for more complicated models. It overcomes the basic disadvantages of the MFA method especially for the higher temperatures, making the resulting phase diagrams comparable with the CVMPA method.

2. The fluctuations calculated by summing up partial series and found to be Gaussian are responsible for a significant decrease in ferro-para transition temperatures when compared to the MFA results. They were also found to play a considerable role in the phenomenon of temperature-induced magnetism. Moreover their behaviour in the vicinity of critical lines (and points) may give some new insights into the investigation of the BEG and similar models.
3. The Gaussian fluctuations behave reasonably in zero and critical temperatures, taking zero and maximum values, respectively.

4. Including both the molecular and the quadrupolar field fluctuations leads to significant changes in the behaviour of the BEG model. Including just one field fluctuations reveals their roles played in certain parts of the phase diagram. Moreover these roles should be more relevant when considering low-dimensional systems.

5. Generalizations of the GFA method can be found to supply information on fluctuation and correlation mechanisms for quantum models (e.g. [21]).

\section{References}

[1] M. Blume, V.J. Emery, R.B. Griffiths, Phys. Rev. A 4, 1071 (1971).

[2] W. Hoston, A.N. Berker, Phys. Rev. Lett. 67, 8 (1991).

[3] A.N. Berker, M. Wortis, Phys. Rev. B 14, 11 (1976).

[4] W. Hoston, A.N. Berker, J. Appl. Phys. 70, 10 (1991).

[5] S. Krinsky, D. Furman, Phys. Rev. B 11, 2602 (1975).

[6] Y.-L. Wang, F. Lee, J.D. Kimmel, Phys. Rev. B 36, 16 (1987).

[7] K. Kasono, I. Ono, Z. Phys. B-Condens. Matter 88, (1992).

[8] A. Rachadi, A. Benyoussef, Phys. Rev. B 69, 064423 (2004).

[9] C.C. Loois, G.T. Barkema, C.M. Smith, Phys. Rev. B 78, 184519 (2008).

[10] A. Rosengren, R. Häggkvist, Phys. Rev. Lett. 63, 660 (1989).

[11] K.G. Chakraborty, J.W. Tucker, Physica A 137, 220 (1986); J. Magn. Magn. Mater. 54, 1349 (1986).

[12] F. Mancini, F.P. Mancini, arXiv:0807.2177v1 (2008); F.P. Mancini, arXiv:0909.5433v1 (2009).

[13] F. Mancini, Eur. Phys. J. B 47, 527 (2005).

[14] J.W. Tucker, J. Magn. Magn. Mater. 71, 27 (1987).

[15] A. Rosengren, S. Lapinskas, Phys. Rev. Lett. 71, 1 (1993); Phys. Rev. B 49, 21 (1994).

[16] J.W. Tucker, T. Balcerzak, M. Gzik, A. Sukiennicki, J. Magn. Magn. Mater. 187, 381 (1998).

[17] Z. Onyszkiewicz, Phys. Lett. 76A, 411 (1980).

[18] W. Rudziński, Z. Onyszkiewicz, Phys. Status Solidi B 163, 267 (1991).

[19] L.S. Borkowski, Z. Jacyna-Onyszkiewicz, Surf. Sci. 603, 27 (2009).

[20] M. Blume, Phys. Rev. 141, 517 (1966).

[21] I.V. Stasyuk, K.V. Tabunshchyk, Cond. Matter Phys. 4, 109 (2001). 